\section{Consumo de sustancias psicoactivas en dos regiones argentinas y su relación con indicadores de pobreza}

\author{
Psychoactive substance use in two regions of \\ Argentina and its relationship to poverty \\ Consumo de substâncias psicoativas em duas \\ regiões argentinas e sua relação com \\ indicadores de pobreza
}

Roberto Ariel Abeldaño ${ }^{1}$ Alicia Ruth Fernández 1 Carla Ap. Arena Ventura 2 Juan Carlos Estario 1

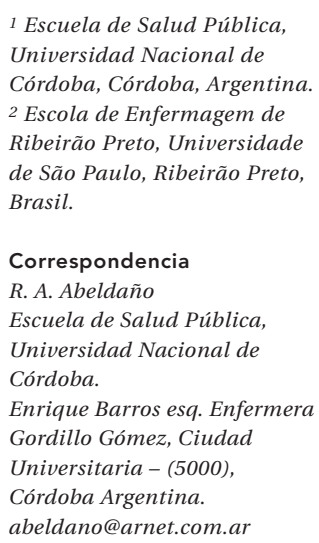

${ }^{1}$ Escuela de Salud Pública, Universidad Nacional de Córdoba, Córdoba, Argentina. 2 Escola de Enfermagem de Ribeirão Preto, Universidade de São Paulo, Ribeirão Preto Brasil.

Correspondencia R. A. Abeldaño

Escuela de Salud Pública, Universidad Nacional de Córdoba.

Enrique Barros esq. Enfermera Gordillo Gómez, Ciudad Universitaria - (5000)

Córdoba Argentina. abeldano@arnet.com.ar

\section{Abstract}

Data were analyzed from the National Survey on Substance Use (ENPreCoSP-2.008) in 6,122 cases to estimate the prevalence of psychoactive substance use and assess the association between unmet basic needs (UBN) and family income and the consumption of psychoactive substances by individuals 18-34 years of age in 10 provinces in Northern Argentina. The study conducted descriptive statistical analysis and logistic regression. Prevalence of lifetime drug use and use in the previous year and previous month were higher for legal versus illegal substances. Consumption was also higher in males. Poverty indicators were significantly associated with the consumption of legal substances. The findings show differences in consumption of psychoactive substances related to gender and poverty.

Street Drugs; Alcohol Drinking; Smoking; Poverty

\section{Resumen}

Se realizó un análisis de base de datos recolectados en la Encuesta Nacional de Prevalencia de Consumo de Sustancias Psicoactivas (ENPreCoSP-2.008) tomando 6.122 casos, con los objetivos de describir las prevalencias de consumo de sustancias psicoactivas; y evaluar la asociación entre la existencia de necesidades básicas insatisfechas y el nivel de ingresos familiares, con el consumo de sustancias psicoactivas en personas entre las edades de 18 a 34 años, residentes en las regiones noroeste (NOA) y noreste (NEA) argentino. Se realizaron análisis descriptivos y regresión logística. Las prevalencias de consumo en la vida, el último año y el último mes fueron mayores en sustancias legales. Así también, el consumo fue mayor en el sexo masculino. Los indicadores de situación de pobreza operaron como factor de riesgo para el consumo de sustancias legales. Los resultados encontrados dan cuenta de situaciones diferenciales en el consumo de sustancias psicoactivas, en relación al género y a las condiciones de pobreza.

Drogas Ilícitas; Consumo de Bebidas Alcohólicas; Tabaquismo; Pobreza 


\section{Introducción}

El consumo de sustancias psicoactivas es un problema complejo, vinculado con factores de muy diversas índoles como: la tolerancia social y falta de observancia de normas y leyes, la disponibilidad de sustancias, la criminalidad y violencia social, el deterioro de vínculos sociales y familiares, las carencias sociales relacionadas con la pobreza y otros factores intrínsecos a los consumidores de esas sustancias 1,2. En consonancia con esto algunos estudios latinoamericanos evidencian que los adolescentes, cuyas familias que viven en situación de pobreza y exclusión social, son vulnerables al consumo de alcohol y otras sustancias psicoactivas 3,4 .

En este sentido, las drogas lícitas e ilícitas además de causar problemas de salud en las personas que las consumen, también ocasionan efectos sociales identificados por algunos autores 5,6. Siendo así, esta problemática constituye a nivel mundial y nacional una preocupación creciente tanto en el ámbito político, social, académico y de la salud, no sólo por las implicaciones que causa en la persona, sino también en el ámbito familiar y social.

En la literatura se aprecia un renovado interés por la investigación de las crecientes y persistentes inequidades en salud y su relación con el consumo de sustancias psicoactivas, de ahí que las recomendaciones de muchos estudios apuntan a ampliar la investigación hacia los factores socioeconómicos que se asocian al consumo de sustancias; como así también en la identificación de los diferenciales sociales en el consumo y sus consecuencias sobre los grupos vulnerables desde la perspectiva de salud pública 7,8,9, incluyendo principalmente a la franja etaria que va desde los 18 a los 34 años de edad, justificándose esto por ser el intervalo de edades que presenta mayores prevalencias de consumo en estudios nacionales anteriores, llevados a cabo por la Secretaría de Programación para la Prevención de la Drogadicción y la lucha contra el Narcotráfico (SEDRONAR) en Argentina 10,11,12.

Entre los antecedentes se puede destacar un estudio desarrollado por un equipo de investigadores estadounidenses, quienes analizaron una encuesta poblacional llevada a cabo en el año 2000, para indagar la relación entre algunos aspectos del capital social con el consumo de drogas y alcohol, encontrando que los adolescentes entre 12 y 17 años que habían reportado niveles medios o altos de capital social, evidenciaron coeficientes de riesgo menores para el consumo de sustancias psicoactivas que los adolescentes que habían reportado niveles bajos de capital social 13. Otra investigación reali- zada en Estados Unidos evaluó el efecto de las diferencias del contexto socioeconómico para la experimentación con cocaína, encontrando una correlación espacial con el incremento de las tasas de experimentación con cocaína en los hogares de comunidades viviendo en condiciones de pobreza 14 .

Investigadores brasileños estudiaron en una muestra de mujeres provenientes de comunidades pobres de Bahía, Brasil, la asociación entre el bajo nivel educativo y el desempleo con comportamientos sexuales de riesgo, al haber intercambiado sexo por dinero para conseguir drogas 15 .

Por medio de una revisión bibliográfica, otros investigadores observaron que el consumo de sustancias psicoactivas es más prevalente en escenarios marcados por el retraso escolar, bajo nivel socioeconómico, bajas relaciones con los padres, entre otros factores 16 .

Autores estadounidenses 17, a través de un análisis longitudinal de las encuestas nacionales de longitudinales, realizadas en Estados Unidos entre los años 1979 y 1998, reportaron incidencias diferenciales en el consumo, como así también patrones diferenciales en la cesación del consumo de cocaína y de marihuana, a partir de la formación de disparidades socioeconómicas, relacionadas con el nivel educativo de la población.

En Argentina, diversos estudios asociaron la condición de pobreza con el consumo de sustancias psicoactivas en jóvenes de las regiones Pampeana, Noroeste y Noreste del país 4; y edades de inicio más precoces para el consumo de tabaco en estratos socioeconómicos bajos 18 . Por su parte, el organismo oficial SEDRONAR ha publicado las tendencias de consumo de sustancias entre los años 2004 y 2010 en población de 15 a 65 años, informando prevalencias de $50 \%$ y $70 \%$ en el consumo de alcohol y tabaco respectivamente, mientras que en el caso de las sustancias ilícitas las prevalencias reportadas por dicho estudio fueron entre el $7,8 \%$ y $10,8 \% 12$.

En base a las evidencias encontradas, se justifica este trabajo debido a que se estima que un mayor conocimiento de estos aspectos puede ayudar a los tomadores de decisiones en el diseño de políticas para la prevención en grupos en condiciones de vulnerabilidad, pobreza o exclusión. Considerando este escenario, los objetivos de este trabajo fueron (1) describir las prevalencias de consumo de sustancias psicoactivas; y (2) evaluar la asociación entre la existencia de necesidades básicas insatisfechas y el nivel de ingresos familiares, con el consumo de sustancias psicoactivas en personas entre las edades de 18 a 34 años, en las regiones noroeste y noreste argentino. 


\section{Material y método}

Se realizó un análisis de una base de datos, de fuente secundaria de acceso público en el sitio web del Instituto Nacional de Estadísticas y Censos (INDEC) de la Argentina, recolectados por la misma institución, a través de la Encuesta $\mathrm{Na}$ cional de Prevalencia de Consumo de Sustancias Psicoactivas (ENPreCoSP-2.008), realizada en un total de 34.203 individuos de 24 jurisdicciones en el año 2008.

El diseño de la muestra incluyó procedimientos de muestreo probabilístico de áreas y estratificado, de acuerdo a tamaño de la aglomeración en función de viviendas, jurisdicción del país y ubicación geográfica, datos de actividad económica y tasa de crecimiento intercensal; contemplando la selección de personas en viviendas particulares del país, ubicadas en aglomeraciones con al menos 5.000 habitantes. El plan de muestreo comprendió en la primera etapa el muestreo de aglomeraciones (una localidad o conjunto de localidades que por continuidad de edificaciones y calles configuran una misma unidad urbana); en una segunda etapa el muestreo de radios censales (unidades menores conformadas por un conjunto de manzanas de alrededor de 300 viviendas); en una tercera etapa fueron seleccionadas de cada área, una muestra de viviendas en forma sistemática. Dentro de cada una de esas viviendas, fueron relevados todos los hogares encontrados en las mismas, y dentro de cada hogar, fue seleccionado al azar un individuo de 16 a 65 años para responder al cuestionario específico de consumo de sustancias psicoactivas, siendo ésta la cuarta y última etapa de muestreo 19.

El índice de no respuesta fue del 14,9\%, en hogares donde no se logró concretar la entrevista, mientras que el índice de no respuesta individual fue del $3,7 \%$.

El INDEC aplicó factores de expansión, corrigiendo posibles sesgos a cada etapa de selección: aglomerado, área en cada aglomerado y vivienda dentro de cada área; el factor de corrección por no respuesta fue definido como el cociente entre las viviendas que responden, más las que no responden, teniendo al menos un hogar, dividido por las viviendas respuestas. En el presente trabajo, el ajuste de los ponderadores se realizó en función de la muestra, considerando: pesos = (Pondera / N) *n.

El instrumento aplicado por el INDEC 19 constó de dos bloques estructurales: el primer bloque trataba sobre los aspectos sociodemográficos de todos los individuos que conforman el hogar, así como la estructura habitacional/material de la vivienda del hogar; el segundo bloque trataba sobre variables que involucraron aspec- tos sociodemográficos complementarios al primer bloque, variables educativas, condición de actividad, variables sobre el estado de salud en términos generales y variables sobre el consumo de sustancias psicoactivas que involucraron al integrante seleccionado en la última etapa del muestreo.

La muestra que se consideró para el análisis del presente estudio estuvo conformada por los 6.122 individuos entre 18 a 34 años, correspondientes a las regiones estadísticas del Noreste y Noroeste, y que comprendieron a las provincias de: Corrientes, Chaco, Formosa, Jujuy, La Rioja, Misiones, Salta, Santiago del Estero, Tucumán. Del total de la base de datos fueron seleccionadas un conjunto de variables relacionadas a las prevalencias de consumo de sustancias psicoactivas y a las condiciones socioeconómicas de la población. Las variables analizadas fueron las siguientes:

- Jurisdicción del país: Corrientes, Chaco, Formosa, Jujuy, La Rioja, Misiones, Salta, Santiago del Estero, Tucumán.

- Existencia de necesidades básicas insatisfechas (NBI): las NBI fueron definidas de acuerdo con la metodología utilizada por INDEC en $L a$ Pobreza en la Argentina 20; considerando como hogar pobre a los que presentan al menos uno de los siguientes indicadores de privación: hogares en condiciones de hacinamiento, hogares en viviendas inadecuadas, hogares en viviendas sin servicios básicos y hogares con absentismo escolar.

- Nivel de ingresos del hogar: rango de ingreso total mensual del hogar en pesos.

- Características de la persona encuestada: sexo, edad, situación conyugal, nivel de instrucción, cobertura de salud y condición de actividad.

- Prevalencia de consumo en la vida, en el año y en el mes de sustancias psicoactivas. Las sustancias fueron operacionalizadas como: legales (tabaco, alcohol, inhalables, fármacos) e ilegales (marihuana, cocaína, pasta base, éxtasis).

Se realizó un análisis exploratorio de frecuencias para variables categóricas, y medidas de resumen para variables mensurables. Se definió un modelo de regresión logística para la identificación de factores de riesgo sobre la variable dependiente prevalencia de consumo de sustancias psicoactivas legales, con un intervalo del 95\% de confianza y un valor de $\mathrm{p}<0,05$. Los datos se procesaron con software SPSS 18 (SPSS Inc., Chicago, Estados Unidos).

En relación a las consideraciones éticas, la base de datos de la EnPreCoSP-2.008 es de acceso público a través del sitio web del INDEC. El proyecto que sustentó al presente estudio obtuvo 
la aprobación del comité de ética del Hospital Nacional de Clínicas de la ciudad de Córdoba, Argentina.

\section{Resultados}

La muestra total analizada en el presente trabajo quedó constituida por 6.122 personas, residentes en las 10 provincias del noroeste y noreste argentino, el rango de edad analizado fue de 18 a 34 años, con una media de 26,06 años ( $\mathrm{DE} \pm 4,878$ ). La distribución porcentual de la población en las 10 provincias del norte argentino osciló entre el $8,48 \%$ (en la provincia de Santiago del Estero) y $10,86 \%$ (en el caso de la provincia de Salta). El $54,67 \%$ fueron mujeres, mientras que el $45,33 \%$ de los encuestados fueron varones. Las edades se distribuyeron el 40,40\% entre los 18 y 24 años, mientras que el restante $59,60 \%$ entre los 25 y 34 años. En la Tabla 1 se pueden observar las demás características demográficas analizadas en el presente trabajo.

Entre las variables socioeconómicas, se observó que la condición de actividad predominante fue la categoría de ocupado, con el $62,72 \%$ de las personas encuestadas, mientras que el 6,03\% de la muestra se encontraba en situación de desocupación al momento de la recolección de datos. El 51,62\% de la población declaró tener un ingreso familiar total mensual menor a $\$ 1.000$, seguido de un $28,81 \%$ que declaró tener ingresos entre \$1.001 y \$2.000. En relación con los indicadores de NBI analizados, en el 16,81\% de la población se observó la ausencia de servicio básico de eliminación de excretas (baño en la vivienda), siendo ésta la necesidad que se presentó con mayor frecuencia entre la población estudiada. Así también, el $77,15 \%$ de la población bajo análisis no se encontraba en situación de pobreza en el momento del estudio, dado que no presentaba ningún indicador de NBI, mientras que el $17,71 \%$ se encontraba en situación de pobreza al acumular al menos un indicador de NBI, de acuerdo a los criterios demográficos y económicos que el INDEC utiliza para la medición de la situación de pobreza (Tabla 2).

En lo que respecta a las prevalencias de consumo de sustancias psicoactivas en la muestra analizada, el 78,32\% manifestó haber consumido algún tipo de sustancia legal "alguna vez en la vida", mientras que el $69,16 \%$ declaró haber consumido alguna sustancia psicoactiva de uso legal en el último año; y el 54,21\% de la muestra había consumido alguna de estas sustancias (alcohol, tabaco, inhalables o fármacos) dentro de los últimos 30 días. Entre este grupo de sustancias, el alcohol fue la sustancia que presentó la mayor prevalencia de uso en la vida, en el año y en el mes $(73,24 \%, 63 \%$ y $42,37 \%$ respectivamente), seguido del tabaco $(48,68 \%, 35,82 \%$ y $31,51 \%$ respectivamente).

En relación con las sustancias de uso ilegal (marihuana, cocaína, pasta base y éxtasis), la prevalencia de uso de éstas fue de $5,08 \%$ en la vida, de $1,16 \%$ en el último año, y de $0,47 \%$ en el último mes. Aquí, la marihuana fue la que reportó la mayor prevalencia con un $4,85 \%$ de uso en la vida. En la Tabla 3 pueden observarse las prevalencias de cada sustancia analizada.

En cuanto al consumo de sustancias psicoactivas, según los rangos de edad analizados (de 18 a 24 años y de 25 a 34 años), se observaron valores similares en ambos grupos de edad: consumo de sustancias legales en la vida de $78,57 \%$ en el grupo de menor edad, y de $78,16 \%$ en el grupo de mayor edad ( $p=0,702)$; consumo en el último año de $69,59 \%$ y de $68,87 \%$ ( $\mathrm{p}=0,862$ ) para cada grupo respectivamente; y de $54,51 \%$ y $54,01 \%(p=0,548)$ en cada grupo de edad para el consumo en el último mes. En el caso de las sustancias de uso ilegal, las prevalencias mostraron comportamientos similares en ambos grupos de edad.

Al relacionar con el sexo, se observaron mayores prevalencias en el sexo masculino que en el sexo femenino, tanto en el consumo de sustancias legales e ilegales $(p=0,0001)$.

Para analizar la presencia de indicadores de NBI entre las personas que consumieron algún tipo de sustancia psicoactiva, se seleccionaron los casos con prevalencias en la vida, último año y último mes, observándose las mayores frecuencias en las personas que no presentaron ningún indicador de necesidades básicas insatisfechas, a su vez se destaca que entre las personas que sí presentaron necesidades básicas insatisfechas la distribución fue mayor entre quienes acumularon sólo un indicador de NBI. En la misma selección de casos, se evaluó el consumo de sustancias psicoactivas según el nivel de ingresos de la muestra estudiada, en este grupo de personas se observó que el mayor consumo de sustancias legales e ilegales estuvo presente entre las personas que declararon tener un ingreso total familiar mensual de $\$ 1$ a $\$ 2.000$, seguido por la prevalencia de consumo de las personas que declararon un ingreso total familiar mensual entre $\$ 2.001$ y $\$ 4.000$ (Tabla 4).

Con el propósito de evaluar los coeficientes de oportunidad sobre la prevalencia de consumo de sustancias psicoactivas legales, se planteó un modelo de regresión logística binaria, introduciendo como variables explicativas a: NBI (mensurable), sexo (categórica), edad (mensurable), y línea de pobreza (categórica). 
Tabla 1

Distribución de las personas encuestadas según características demográficas. Regiones noroeste y noreste argentino. Encuesta Nacional de Prevalencia de Consumo de Sustancias Psicoactivas-2.008 ( $N=6.122$ ).

\begin{tabular}{|c|c|c|}
\hline Variables/Categorías & $n$ & $\%$ \\
\hline \multicolumn{3}{|l|}{ Jurisdicción del país } \\
\hline Catamarca & 621 & 10,14 \\
\hline Corrientes & 584 & 9,54 \\
\hline Chaco & 626 & 10,23 \\
\hline Formosa & 528 & 8,62 \\
\hline Jujuy & 653 & 10,67 \\
\hline La Rioja & 664 & 10,85 \\
\hline Misiones & 608 & 9,93 \\
\hline Salta & 665 & 10,86 \\
\hline Santiago del Estero & 519 & 8,48 \\
\hline Tucumán & 654 & 10,68 \\
\hline \multicolumn{3}{|l|}{ Sexo } \\
\hline Mujer & 3.347 & 54,67 \\
\hline Varón & 2.775 & 45,33 \\
\hline \multicolumn{3}{|l|}{ Grupo de edad (años) } \\
\hline $18-24$ & 2.473 & 40,40 \\
\hline $25-34$ & 3.649 & 59,60 \\
\hline \multicolumn{3}{|l|}{ Situación conyugal } \\
\hline Unido/a & 1.482 & 24,21 \\
\hline Casado/a & 1.025 & 16,74 \\
\hline Separado/a & 165 & 2,70 \\
\hline Divorciado/a & 25 & 0,41 \\
\hline Viudo/a & 16 & 0,26 \\
\hline Soltero/a & 3.409 & 55,68 \\
\hline \multicolumn{3}{|l|}{ Nivel de instrucción } \\
\hline Sin instrucción & 28 & 0,46 \\
\hline Primario incompleto & 316 & 5,16 \\
\hline Primario completo & 756 & 12,35 \\
\hline Secundario incompleto & 1.307 & 21,35 \\
\hline Secundario completo & 1.460 & 23,85 \\
\hline Terciario o universitario incompleto & 1.617 & 26,41 \\
\hline Terciario o universitario completo y más & 623 & 10,18 \\
\hline Educación especial & 15 & 0,25 \\
\hline \multicolumn{3}{|l|}{ Cobertura de salud * } \\
\hline Sólo plan de salud privado & 344 & 5,62 \\
\hline Plan de salud privado y otro & 111 & 1,81 \\
\hline Sólo obra social & 2.384 & 38,94 \\
\hline Obra social y otro no privado & 116 & 1,89 \\
\hline Sólo servicio de emergencia & 70 & 1,14 \\
\hline Sólo plan o seguro público & 56 & 0,91 \\
\hline Plan o seguro público y servicio de emergencia & 1 & 0,02 \\
\hline No está asociado a nada & 2.940 & 48,02 \\
\hline
\end{tabular}

* Valores perdidos de cobertura de salud: 100 casos. 
Distribución de las personas encuestadas, según condición de actividad, rango de ingresos mensuales totales del hogar y presencia de necesidades básicas insatisfechas. Encuesta Nacional de Prevalencia de Consumo de Sustancias Psicoactivas-2.008 $(N=6.122)$

\begin{tabular}{|c|c|c|}
\hline Variables/Categorías & $\mathrm{N}$ & $\%$ \\
\hline \multicolumn{3}{|l|}{ Condición de actividad } \\
\hline Ocupado & 3.840 & 62,72 \\
\hline Desocupado & 369 & 6,03 \\
\hline Inactivo & 1.913 & 31,25 \\
\hline \multicolumn{3}{|c|}{ Rango del ingreso total mensual del hogar (en Pesos) * } \\
\hline Sin ingresos & 17 & 0,28 \\
\hline $1-1.000$ & 3.160 & 51,62 \\
\hline $1.001-2.000$ & 1.764 & 28,81 \\
\hline $2.001-3.000$ & 534 & 8,72 \\
\hline $3.001-4.000$ & 188 & 3,07 \\
\hline $4.001-5.000$ & 108 & 1,76 \\
\hline $5.001-7.500$ & 40 & 0,65 \\
\hline $7.501-10.000$ & 21 & 0,34 \\
\hline 10.001 y más & 4 & 0,07 \\
\hline \multicolumn{3}{|l|}{ NBI 1 - Vivienda inadecuada } \\
\hline No & 5.847 & 95,51 \\
\hline Sí & 275 & 4,49 \\
\hline \multicolumn{3}{|l|}{ NBI 2 - Hacinamiento } \\
\hline No & 5.714 & 93,34 \\
\hline Sí & 408 & 6,66 \\
\hline \multicolumn{3}{|l|}{ NBI 3 - Hogares sin baño } \\
\hline No & 5.093 & 83,19 \\
\hline Sí & 1.029 & 16,81 \\
\hline \multicolumn{3}{|l|}{ NBI 4 - Educación } \\
\hline No & 6.070 & 99,15 \\
\hline $\mathrm{Si}$ & 52 & 0,85 \\
\hline \multicolumn{3}{|l|}{ NBI total } \\
\hline No acumula ningún indicador & 4.723 & 77,15 \\
\hline Acumula al menos un indicador & 1.084 & 17,71 \\
\hline Acumula 2 indicadores & 265 & 4,33 \\
\hline Acumula 3 indicadores o más & 50 & 0,82 \\
\hline
\end{tabular}

NBI: necesidades básicas insatisfechas.

*Valores perdidos de rango del ingreso total mensual del hogar en pesos: 286 casos.

El modelo de regresión logística sobre la prevalencia de consumo de sustancias psicoactivas legales en el último año $(\mathrm{y}=1)$ versus la "no prevalencia" de esas sustancias $(\mathrm{y}=0)$ resultó significativo en su conjunto $(p=0,001)$. Las variables que podrían aumentar el riesgo de consumo son las NBI acumuladas (OR = 1,275; IC: 1,009-1,612) y el sexo (masculino) $(\mathrm{OR}=3,037$; IC: 2,690 3,428 ), mientras que tener ingresos superiores a la línea de la pobreza podría considerarse como un factor de protección $(\mathrm{OR}=0,731$; IC: 0,621 $0,861)$.
Se observó un comportamiento similar de estos coeficientes sobre la prevalencia de consumo de sustancias psicoactivas legales en el último mes $(\mathrm{y}=1)$ versus la "no prevalencia" $(\mathrm{y}=0)$ (Tabla 5).

\section{Discusión}

Los índices encontrados en este trabajo, en referencia a la situación de pobreza a partir de las necesidades básicas insatisfechas, son coinci- 
Prevalencias de consumo de sustancias psicoactivas, en la vida, en el último año y en el último mes, según tipo de sustancias. Regiones noroeste y noreste argentino. Encuesta Nacional de Prevalencia de Consumo de Sustancias Psicoactivas-2.008 $(\mathrm{N}=6.122)$

\begin{tabular}{lcccccc}
\hline Prevalencias de consumo & \multicolumn{2}{c}{ En la vida } & \multicolumn{2}{c}{ En el último año } & \multicolumn{2}{c}{ En el último mes } \\
& $\mathbf{n}$ & $\%$ & $\mathbf{n}$ & $\%$ & $\mathbf{n}$ & \multicolumn{2}{c}{$\%$} \\
\hline Sustancias legales & 4.795 & 78,32 & 4.234 & 69,16 & 3.319 & 54,21 \\
Alcohol & 4.484 & 73,24 & 3.857 & 63,00 & 2.594 & 42,37 \\
Tabaco & 2.980 & 48,68 & 2.193 & 35,82 & 1.929 & 31,51 \\
Tranquilizantes & 224 & 3,66 & 97 & 1,58 & 43 & 0,70 \\
Estimulantes & 34 & 0,56 & 15 & 0,25 & 3 & 0,05 \\
Inhalables & 46 & 0,75 & 9 & 0,15 & 3 & 0,05 \\
Sustancias ilegales & 311 & 5,08 & 71 & 1,16 & 29 & 0,47 \\
Marihuana & 297 & 4,85 & 64 & 1,05 & 24 & 0,39 \\
Cocaína & 74 & 1,21 & 15 & 0,25 & 6 & 0,10 \\
Pasta base & 22 & 0,36 & 5 & 0,08 & 2 & 0,03 \\
Éxtasis & 11 & 0,18 & 0 & 0,00 & 0 & 0,00 \\
\hline
\end{tabular}

$\underline{\text { Tabla } 4}$

Prevalencias de consumo de sustancias psicoactivas por tipo de sustancias, según rangos de ingresos mensuales totales familiares. Encuesta Nacional de Prevalencia de Consumo de Sustancias Psicoactivas-2.008 ( $\mathrm{N}=5.836$ ).

\begin{tabular}{|c|c|c|c|c|c|}
\hline Categorías de prevalencia & Sin ingresos (\%) & $1-2.000(\%)$ & $2.001-4.000(\%)$ & $4.001-7.500(\%)$ & 7.501 y más (\%) \\
\hline \multicolumn{6}{|l|}{ Sustancias legales } \\
\hline Prevalencia de vida & 0,25 & 79,75 & 12,26 & 2,73 & 0,50 \\
\hline Prevalencia último año & 0,28 & 79,00 & 12,90 & 2,79 & 0,57 \\
\hline Prevalencia último mes & 0,24 & 78,97 & 13,08 & 2,80 & 0,57 \\
\hline \multicolumn{6}{|l|}{ Sustancias ilegales } \\
\hline Prevalencia de vida & 0,32 & 77,81 & 15,43 & 3,22 & 0,64 \\
\hline Prevalencia último mes & 0,00 & 81,69 & 15,49 & 1,41 & 0,00 \\
\hline Prevalencia último año & 0,00 & 82,76 & 13,79 & 3,45 & 0,00 \\
\hline
\end{tabular}

dentes con los hallazgos reportados en Argentina 4,21 y con las estimaciones informada por la Comisión Económica para América Latina y el Caribe (CEPAL), quienes desde la década de 1990 ya exponían que las jurisdicciones del norte del país se encontraban en situación de desventaja socioeconómica con respecto a las jurisdicciones metropolitanas, si se analizan los indicadores multidimensionales de pobreza 22.

Lo observado en relación al consumo de sustancias legales, y en particular ubicando al alcohol y al tabaco como las sustancias psicoactivas de consumo más prevalente, son compatibles con los datos relatados por el Informe Brasileño sobre Drogas (Relatório Brasileiro Sobre Drogas 23). Así también, el mismo informe ubica en orden de jerarquía, a la marihuana y a la cocaína como las dos sustancias de uso ilegal de mayor consumo en Brasil, situación similar a lo observado en la muestra analizada en este trabajo.

De la misma manera, investigadores peruanos encontraron en población universitaria el consumo de marihuana como la sustancia ilegal con mayor prevalencia de vida, aunque aparece allí la pasta básica de cocaína como la segunda sustancia, en términos de prevalencia de consumo, mientras que en el presente trabajo dicha sustancia es la tercera, tras el clorhidrato de cocaína.

Respecto al consumo de sustancias por sexos y por grupos de edades, se obtuvieron mayores prevalencias en personas de sexo masculino, lo 
Coeficientes de odds ratio sobre las prevalencias de consumo de sustancias legales en el último año y en el último mes según necesidades básicas insatisfechas acumuladas, sexo, edad y línea de pobreza. Encuesta Nacional de Prevalencia de Consumo de Sustancias Psicoactivas-2008 ( $N=5.836$ ).

\begin{tabular}{|c|c|c|c|c|c|}
\hline \multirow[t]{2}{*}{ Variables dependientes } & \multirow[t]{2}{*}{ Variables independientes } & \multirow[t]{2}{*}{ Valor de $p$} & \multirow[t]{2}{*}{ OR } & \multicolumn{2}{|c|}{ IC95\% para OR } \\
\hline & & & & Inferior & Superior \\
\hline Prevalencia de consumo & NBI acumuladas & 0,042 & 1,275 & 1,009 & 1,612 \\
\hline en el último año & Sexo & 0,001 & 3,037 & 2,690 & 3,428 \\
\hline \multirow[t]{2}{*}{ (sustancias legales) } & Edad & 0,543 & 0,996 & 0,985 & 1,008 \\
\hline & Línea de pobreza & 0,001 & 0,731 & 0,621 & 0,861 \\
\hline Prevalencia de consumo & NBI acumuladas & 0,028 & 1,278 & 1,027 & 1,590 \\
\hline en el último mes & Sexo & 0,001 & 3,240 & 2,905 & 3,614 \\
\hline \multirow[t]{2}{*}{ (Sustancias legales) } & Edad & 0,985 & 1,000 & 0,989 & 1,011 \\
\hline & Línea de pobreza & 0,030 & 0,839 & 0,716 & 0,983 \\
\hline
\end{tabular}

IC95\%: intervalo del 95\% de confianza; NBI: necesidades básicas insatisfechas; OR: odds ratio.

cual acuerda con lo notificado por el mismo informe brasileño sobre drogas. A su vez, otros investigadores peruanos observaron prevalencias con diferencias entre los grupos de edades de 18 a 24 y de 25 a 34 años 4,24 .

Los hallazgos en el consumo de sustancias entre los sectores socioeconómicos empobrecidos son coincidentes con otros estudios que asociaron a las condiciones de pobreza - medida en NB - como factor de riesgo para el consumo excesivo de alcohol en jóvenes argentinos de 16 a 24 años 4; como así también con investigaciones que relevaron mayores prevalencias de consumo de alcohol y tabaco en personas viviendo en sectores geográficos urbanos de Tanzania, con condiciones económicas de pobreza ${ }^{8}$. El estudio de caso de Miech \& Chilcoat 17 sobre la población estadounidense en los años 90 mostró que las desigualdades socioeconómicas, generadas desde las condiciones educativas, jugaron un papel influyente en el consumo y en la cesación uso de cocaína y marihuana. Un lectura similar, en relación a las condiciones de pobreza, fue observada en Francia sobre una muestra de 6.216 personas mayores de 16 años, donde encontraron patrones de consumo de tabaco, abuso de alcohol y uso frecuente de drogas psicotrópicas asociados con el mayor nivel de privación material del hogar 25 .

Todos estos reportes son disímiles a lo observado en el estudio de Sanchez et al. 26, donde los jóvenes estudiantes de escuelas privadas de las ciudades capitales de Brasil podrían evidenciar un riesgo aumentado para el consumo excesivo de alcohol en esos jóvenes, debido precisamente a su alta condición socioeconómica, demostran- do de alguna manera la necesidad de disponibilidad de recursos económicos para ese patrón en el consumo de alcohol.

De acuerdo a lo publicado por la Comisión sobre Determinantes Sociales de la Salud de la Organización Mundial de la Salud, se relata un aumento en los indicadores de consumo de alcohol y otras sustancias psicoactivas, en coherencia con los fenómenos de transición demo-epidemiológica que acompañan a las modificaciones en los problemas de salud de la población, en particular entre las comunidades pobres de los entornos urbanos. En este sentido, es importante el aporte académico en la evaluación de las repercusiones de las situaciones de desigualdad social que tienen en la salud de las poblaciones. En consecuencia, se considera que para reducir la prevalencia del consumo de sustancia psicoactivas es necesario elaborar estrategias integrales, centradas en la reducción de los determinantes sociales de la salud 27 .

\section{Conclusiones}

Los resultados encontrados dan cuenta de situaciones diferenciales en el consumo de sustancias psicoactivas, en relación al género y a las condiciones de pobreza en las regiones del norte argentino. Resulta pertinente recomendar un trabajo sostenido en políticas para la reducción de las inequidades sanitarias y las desigualdades en las condiciones de vida que para reducir el consumo de sustancias psicoactivas. 


\section{Resumo}

Realizou-se uma análise de base de dados coletados durante $a$ Encuesta Nacional de Prevalencia de Consumo de Sustancias Psicoactivas (ENPreCoSP-2.008) tomando 6.122 casos, com objetivos de descrever as prevalências de consumo de substâncias psicoactivas, $e$ avaliar a associação entre a existência de necessidades básicas insatisfeitas e o nível de renda familiar, com o consumo de substâncias psicoactivas em pessoas de 18 a 34 anos, residentes nas regiões noroeste (NOA) $e$ nordeste (NEA) da Argentina. Realizaram-se análises descritivas e regressão logística. As prevalências de consumo durante a vida, o último ano e o último mês foram maiores entre as substâncias legais. O consumo também foi maior no sexo masculino. Os indicadores da situação de pobreza operaram como fator de risco para o consumo de substâncias legais. Os resultados encontrados mostram situações diferenciais no consumo de substâncias psicoativas, em relação ao gênero e as condições de pobreza.

Drogas Ilícitas; Consumo de Bebidas Alcoólicas; Tabagismo; Pobreza

\section{Colaboradores}

R. A. Abeldaño contribuyó en la concepción y el diseño, adquisición de datos, análisis e interpretación de datos, en la redacción del artículo y la aprobación final de la versión publicada. A. R. Fernández, C. Ap. A. Ventura y J. C. Estario contribuyeron a la redacción del artículo y la revisión crítica de su contenido.

\section{Agradecimientos}

Escola de Enfermagem de Ribeirão Preto de la Universidade de São Paulo, Ribeirão Preto, Brasil.

\section{Referencias}

1. Ripple C, Luthar S. Familial factors in illicit drug abuse: an interdisciplinary perspective. Am J Drug Alcohol Abuse 1996; 22:147-72.

2. Díaz Negrete B, García-Aurrecoechea R. Factores psicosociales de riesgo de consumo de drogas ilícitas en una muestra de estudiantes mexicanos de educación media. Rev Panam Salud Pública 2008; 24:223-32.

3. Martins M, Santos M, Pillon S. Low-income families' perceptions on the use of drugs by one of their members. Rev Latinoam Enferm 2008; 16:293-8.

4. Acosta L, Fernández R, Pillon S. Factores sociales para el uso de alcohol en adolescentes y jóvenes. Rev Latinoam Enferm 2011; 19:771-81.

5. Leiva Diaz M, Pereira Vasters G, Lobo da Costa M. Caracterización de estudiantes de la carrera de enfermería sobre consumo de drogas lícitas e ilícitas. Rev Latinoam Enferm 2010; 18:565-72.

6. Fairbairn N, Small W, Van Borek N, Wood E, Kerr T. Social structural factors that shape assisted injecting practices among injection drug users in Vancouver, Canada: a qualitative study. Harm Reduct J 2010; 7:20.
7. Roberts E, Friedman S, Brady J, Pouget E, Tempalski B, Galea S. Environmental conditions, political economy, and rates of injection drug use in large US metropolitan areas 1992-2002. Drug Alcohol Depend 2010; 106:142.

8. Mbatia J, Jenkins R, Singleton N, White B. Prevalence of alcohol consumption and hazardous drinking, tobacco and drug use in urban Tanzania, and their associated risk factors. Int J Environ Res Public Health 2009; 6:1991-2006.

9. Lloyd-Smith E, Wood E, Li K, Montaner J, Kerr T. Incidence and determinants of initiation into cocaine injection and correlates of frequent cocaine injectors. Drug Alcohol Depend 2009; 99:176-82.

10. Observatorio Argentino de Drogas. Informe de resultados: estudio nacional en población de 12 a 65 años, sobre consumo de sustancias psicoactivas, Argentina 2006. Buenos Aires: Secretaría de Programación para la Prevención de la Drogadicción y la Lucha contra el Narcotráfico; 2007. 
11. Míguez H. Resumen de la Encuesta Epidemiológica sobre Prevalencia de Consumo de sustancias psicoactivas en Argentina - 1999. Buenos Aires: Secretaría de Programación para la Prevención de la Drogadicción y la Lucha contra el Narcotráfico; 1999.

12. Observatorio Argentino de Drogas. Tendencias en el consumo de sustancias psicoactivas en Argentina 2004-2010, población de 16 a 65 años. Buenos Aires: Secretaría de Programación para la Preven ción de la Drogadicción y la Lucha contra el Narcotráfico; 2011.

13. Winstanleya EL, Steinwachs DM, Ensmingerc ME, Latkinc CA, Stitzera ML, Olsen Y. The association of self-reported neighborhood disorganization and social capital with adolescent alcohol and drug use, dependence, and access to treatment. Drug Alcohol Depend 2008; 92:173-82.

14. Petronis KR, Anthony JC. A different kind of contextual effect: geographical clustering of cocaine incidence in the USA. J Epidemiol Community Health 2003; 57:893-900.

15. Nunes CLX, Andrade T, Galvão-Castro B, Bastos FI, Reingold A. Assessing risk behaviors and prevalence of sexually transmitted and blood-borne infections among female crack cocaine users in Salvador - Bahia, Brazil. Braz J Infect Dis 2007; 11:561-6.

16. Duailibi LB, Ribeiro M, Laranjeira R. Profile of cocaine and crack users in Brazil. Cad Saúde Pública 2008; 24 Suppl 4:S545-57.

17. Miech R, Chilcoat H. The formation of a socioeconomic disparity: a case study of cocaine and marijuana use in the 1990s. Am J Prev Med 2007; 32(6 Suppl):S171-6.

18. Paulone I, Candioti CA. Consumo de sustancias adictivas en adolescentes escolarizados. Arch Argent Pediatr 2006; 104:227-33.

19. Instituto Nacional de Estadísticas y Censos. Encuesta nacional sobre prevalencia de consumo de sustancias psicoactivas (INDEC EnPreCoSP 2008). Documento para la utilización de base de datos usuario. Buenos Aires: Instituto Nacional de Estadísticas y Censos/Ministerio de Salud de la Nación; 2009
20. Instituto Nacional de Estadísticas y Censos. La pobreza en la Argentina. Buenos Aires: Instituto Nacional de Estadísticas y Censos; 1984. (Serie Estudios INDEC, 1).

21. Aguilar M. Las defunciones por enfermedades infecciosas y parasitarias en relación con las condiciones sanitarias de la población argentina en el 2000. Una aproximación a la articulación aguaenfermedad. In: VIII Jornadas Argentinas de Estudios de Población de la Asociación de Estudios de Población de la Argentina. Tandil: Asociación de Estudios de Población de la Argentina; 2005.

22. Hicks N. An analysis of the index of unsatisfied basic needs (NBI) of Argentina with suggestions for improvements. In: La Medición de la Pobreza: Métodos y Aplicaciones. Taller 5. Santiago: Comisión Económica para América Latina y el Caribe; 2000.

23. Duarte PCAV, Stempliuk VA, Barroso LP, organizadores. Relatório brasileiro sobre drogas. Brasília: Secretaria Nacional de Políticas sobre Drogas; 2009.

24. Zárate M, Zavaleta A, Danjoy D, Chanamé E, Prochazka R, Salas M, et al. Prácticas de consumo de tabaco y otras drogas en estudiantes de ciencias de la salud de una universidad privada de Lima, Perú. Invest Educ Enferm 2006; 24:72-81.

25. Baumann M, Spitz E, Guillemin F, Ravaud JF, Choquet M, Falissard B, et al. Associations of social and material deprivation with tobacco, alcohol, and psychotropic drug use, and gender: a populationbased study. Int J Health Geogr 2007; 6:50.

26. Sanchez ZM, Locatelli DP, Noto AR, Martins SS Binge drinking among Brazilian students: a gradient of association with socioeconomic status in five geo-economic regions. Drug Alcohol Depend 2012; 127:87-93.

27. Organización Mundial de la Salud; Organización Panamericana de la Salud. Subsanar las desigualdades en una generación. Resumen Analítico del Informe Final de la Comisión sobre Determinan tes Sociales de la Salud. Geneva: Organización Mundial de la Salud; 2008

Recibido el 21/Ago/2012

Versión final presentada el 22/Dic/2012

Aprobado el 22/Ene/2013 